\title{
Proteomic Identification of Novel Plasma Biomarkers and Pathobiologic Pathways in Alcoholic Acute Pancreatitis
}

\author{
Richard T. Waldron ${ }^{1 *}$, Aurelia Lugea ${ }^{1}$, Aiste Gulla ${ }^{2,3}$ and Stephen J. Pandol ${ }^{1}$ \\ ${ }^{1}$ Pancreatic Research Group, Department of Medicine, Cedars-Sinai Medical Center, Los Angeles, CA, United States, \\ ${ }^{2}$ Department of Surgery, Georgetown University Hospital, Washington, DC, United States, ${ }^{3}$ Department of Surgery, Vilnius \\ University Hospital Santaros Clinics, Faculty of Medicine, Vilnius University, Vilnius, Lithuania
}

\section{OPEN ACCESS}

Edited by:

Steven Dooley,

Medical Faculty Mannheim,

Heidelberg University, Germany

Reviewed by:

Sarbjeet Makkar,

Washington University in St. Louis,

United States

Frank Tacke,

Uniklinik RWTH Aachen, Germany

Michael Chvanov,

University of Liverpool,

United Kingdom

Daniel Globisch,

Uppsala University, Sweden

*Correspondence:

Richard T. Waldron

waldronr@cshs.org

Specialty section:

This article was submitted to

Gastrointestinal Sciences,

a section of the journal

Frontiers in Physiology

Received: 12 May 2018 Accepted: 13 August 2018

Published: 30 August 2018

Citation:

Waldron RT, Lugea A, Gulla A and

Pandol SJ (2018) Proteomic

Identification of Novel Plasma

Biomarkers and Pathobiologic

Pathways in Alcoholic Acute

Pancreatitis. Front. Physiol. 9:1215.

doi: 10.3389/fphys.2018.01215
Acute pancreatitis (AP) is a painful and potentially life-threatening disorder with the potential for therapeutic interventions. Biomarkers that characterize cases by severity and pathogenic mechanisms involved are not yet available but needed for the implementation of rational therapies. Here, we used shotgun proteomics to obtain information from plasma samples about local and systemic pathologies taking place during cases of alcoholic AP. Plasma was obtained at Kaunas University of Medicine Hospital (Lithuania) from 12 AP patients of alcohol related etiology (median age of 40) within $24 \mathrm{~h}$ of presentation, and 12 age-matched, healthy controls. Patients entered into the study had moderately severe AP with the following characteristics: mean blood lactate dehydrogenase level of $1127 \mathrm{mg} / \mathrm{dl}$; median APACHEll score of 5.5 and mean IMRIE score of 3.5. For proteomic analysis, less-abundant proteins in plasma samples were enriched using Top 12 abundant protein depletion columns. Further processing was performed by a modified filter-assisted sample preparation combined with tandem mass tag labeling for quantitation. Samples were analyzed using an Orbitrap Elite mass spectrometer for high resolution liquid chromatography-tandem mass spectrometry (LC-MS/MS). Our analysis revealed 31 proteins that exhibited significant 1.5-fold or higher increases in the AP compared to control patients, and six that were significantly decreased. Gene ontology analysis indicated a strong correlation with exosomal origin in the elevated proteins, with 29/31 (93.5\%) associated with this extracellularly-secreted compartment. Elevated proteins included established and proposed biomarkers of AP including C-reactive protein, LPS-binding protein, intercellular adhesion molecule-1, and von Willebrand factor, as well as several novel potential biomarkers. These results provide the methodology for proteomic analysis of plasma samples to discover novel biomarkers that characterize pancreatitis cases by pathogenic mechanism as well as disease activity at an early stage that is highly informative for routine clinical practice and clinical trials.

Keywords: acute pancreatitis, biomarkers, C-reactive protein, ethanol, Orbitrap Elite, proteomic, severity, transketolase 


\section{INTRODUCTION}

Acute pancreatitis is one of the most common diseases of the gastrointestinal tract, leading to tremendous emotional, physical, and financial human burden (Papachristou et al., 2010). In the United States, in 2009, AP was the most common gastroenterology discharge diagnosis with a cost of 2.6 billion dollars. Recent studies show the incidence of AP varies between 4.9 and 73.4 cases per 100,000 worldwide (Tenner et al., 2013).

Alcohol consumption has been identified as a primary cause of both acute and chronic pancreatitis in most developed countries. About one-third of AP in the United States is alcohol-related, being more common in men, and the other most common cause is gallstones (Dufour and Adamson, 2003). The relative contribution of heavy alcohol consumption is likely to be greater than gallstones for RAP, although this may vary based on the demographic distribution. In Lithuania, as elsewhere in the world, the detrimental effects of alcohol consumption on health are of concern (Klumbiene et al., 2012). Alcohol does not cause pancreatitis by itself but increases the susceptibility to pancreatitis by affecting pancreatic physiology at multiple levels, of which the most important is its ability to sensitize the pancreas to other insults (Aframian et al., 1996; Pandol et al., 1999). One such cofactor is smoking, which can also increase the risk of pancreatitis independently (Andriulli et al., 2010; Greer et al., 2015). Genetic susceptibility can explain an increased risk of pancreatitis in some individuals who also drink.

Regardless of the environmental factors that accompany and modify alcohol drinkers, AP related to alcohol tends to exhibit significant organ damage and can be among the severe cases with systemic inflammatory response syndrome (SIRS) and organ failure involving the pulmonary, cardiovascular, and renal systems (Ignatavicius et al., 2017). Single and combination treatments to combat disorders in these organs have not been developed for AP. Thus, early biomarkers of impending SIRS, MODS, or infected necrosis in AP would be especially valuable.

\footnotetext{
Abbreviations: ACN, acetonitrile; AGT, angiotensinogen; ALB, serum albumin precursor; AP, acute pancreatitis; APACHE, Acute Physiology and Chronic Health Examination; BCA, bicinchoninic acid; $\mathrm{C}$ 1, complement $\mathrm{C1}$; $\mathrm{C} 9$, complement C9; CRP, C-reactive protein; ERCP, endoscopic retrograde cholangiopancreatography; ESCRT, endosomal sorting complex required for transport; F8, coagulation factor VIII; FA, formic acid; FGA, fibrinogen alpha chain; FGB, fibrinogen beta chain precursor; FGG, fibrinogen gamma chain precursor; GCR, glucocorticoid receptor; IAP, intra-abdominal pressure; IL1, interleukin-1; IL6, interleukin-6; IMRIE, modified Glasgow Imrie severity criteria for acute pancreatitis; IPA, ingenuity pathway analysis; ITIH3, inter-alpha-trypsin inhibitor heavy chain 3; LBP, lipopolysaccharide binding protein; LC-MS/MS, liquid chromatographytandem mass spectrometry; LDH, lactate dehydrogenase; LPS binding protein, lipopolysaccharide binding protein; LRG1, leucine-rich alpha-2-glycoprotein 1; MODS, multiple organ dysfunction syndrome; NFIL6, nuclear factor for IL-6 expression; ORM, alpha-1-acid glycoprotein 1; OSM, oncostatin M; RAP, recurrent acute pancreatitis; RT, room temperature; S100A8, S100 calcium-binding protein A8; SAA, serum amyloid A; SCX, strong cation exchange; SDS-PAGE, sodium dodecylsulfate-polyacrylamide gel electrophoresis; SERPINA1, alpha-1-proteinase inhibitor; SERPINA3, alpha-1-antichymotrypsin; SERPING1, plasma protease C1 inhibitor precursor; SIRS, systemic inflammatory response syndrome; SPARCL1, secreted protein, acidic and rich in cysteine-like 1; TBS-T, Tris-buffered saline supplemented with $0.075 \%$ Tween-20; TEAB, triethylammonium bicarbonate; TF, transferrin; TKT, transketolase; TMT, tandem mass tag; TNF, tumor necrosis factor; vWF, von Willebrand factor.
}

Scoring systems such as the Ranson's, APACHE-II, and Imrie systems have been used to help predict the severity level and course of disease, but current trends have emphasized a desire in the field to simplify these systems while retaining their predictive performance (Papachristou et al., 2010; Wu et al., 2017). Many stand-alone biomarkers have been proposed and evaluated over the years, but very few have come into routine clinical practice, either due to insufficient specificity or selectivity in the biomarker or because inexpensive tests are not readily available. CRP, as well as IL- 6 have been found highly specific and selective, and an inexpensive test has come into use for CRP (Meher et al., 2015). This marker typically peaks at $\sim 72 \mathrm{~h}$ after the onset of clinical AP and then subsides in mild, but not in severe cases. Thus, CRP levels above $\sim 250 \mathrm{~g} / \mathrm{L}$ indicate the presence of a systemic inflammatory process such as AP. Improved measures that evaluate the systemic effects of the disease and identify targets for therapeutics are needed. Some experimental therapeutic approaches are already under study and may prove beneficial, but we do not have measures to determine pathways they would affect during the disease. The ongoing development of such measures is necessary, as these will need to be applied to personalized treatment approaches.

Here, we performed a quantitative proteomic technique with plasma proteins depleted of 12 abundant proteins to improve coverage and digested with trypsin into peptides, which were labeled with amino group-reactive tandem mass tags (TMT) to facilitate quantitative determination of relative expression levels in AP patients versus controls. Here, we report several proteins significantly elevated or decreased in the AP compared to the control subjects. Our findings substantiate the recent development of CRP as a valid biomarker of AP that is superior to more classic biomarkers such as pancreatic lipase. While our data specifically adds validity to the use of CRP as a biomarker of alcoholic AP, they also strongly implicate acute phase proteins in general, as 16 distinct "positive acute phase" proteins (SAA, ORM, AGT, LBP, CRP, FGA, FGB, FGG, SERPINA3, F8, SERPINA1, C9, C1, ITIH3, vWF, and SERPING1), and two "negative acute phase" proteins (Ritchie et al., 1999) (ALB and TF) emerged in our list of proteins elevated and decreased, respectively in alcoholic AP. In addition, several proteins significantly elevated or decreased in AP patients that do not correspond to the acute phase response may represent novel AP biomarkers. Using our list of significantly altered proteins in plasma, we also performed IPA to determine pathobiologic regulatory pathways in AP patients.

\section{MATERIALS AND METHODS}

\section{Study Design and Patient Population}

These samples arise from a prospective observational study conducted at the Department of Surgery at Kaunas University of Medicine Hospital (Lithuania). Patients admitted during 20062007 with a diagnosis of AP, history of significant alcohol intake and onset of the disease within last $72 \mathrm{~h}$ were included in this study (Dambrauskas et al., 2010). Plasma from healthy controls was obtained during the same dates by the medical team 
using similar procedures used in patients. The Regional Ethics Committee approved the study (Protocol Nos. BE-2-47 and P1$113 / 2005$ ) and all the patients and healthy controls provided written informed consent. The AP diagnosis was established on the basis of acute abdominal pain consistent with AP; and at least threefold elevated levels of serum amylase or typical radiological findings of AP. A contrast-enhanced CT scan was performed on days 4 to 7 after onset of the disease to determine the presence of pancreatic necrosis. Patients with underlying chronic pancreatitis and patients with AP referred to the hospital from other institutions after management for more than 3 days were excluded from this study. Age- and sex-matched healthy subjects $(n=18)$ without previous medical history were enrolled as controls. Volunteers were excluded from participation on the basis of the presence of any illness or any recent surgical procedure.

\section{Composite Clinical Scores}

For each participant, the APACHE II scores were those calculated on the day of admission. Similarly, the Marshall multiple organ dysfunction syndrome (MODS) score reported was that calculated initially. Severity was assessed according to the clinical course and clinical scores (APACHE II $>7$; Imrie-Glasgow $>2$; MODS > 2). Clinical data relating to the severity of disease, development of organ dysfunction and/or septic complications were prospectively collected in a standardized fashion. The plasma sample Serum CRP and white blood cells were measured to assess the inflammatory burden of the cohort.

\section{Sample Acquisition and Handling}

Peripheral blood samples from patients were drawn on admission to the hospital and, after centrifugation, plasma samples were stored at $-80^{\circ} \mathrm{C}$ until analysis. The blood samples of control group underwent a similar process. Plasma samples were transferred to Cedars-Sinai Medical Center, Los Angeles, CA, United States where the proteomic analysis was performed. All the procedures were approved by the Cedars-Sinai Medical Center IRB, under protocol Pro00048082.

\section{Plasma Depletion of Abundant Proteins}

Selected AP and control plasma samples were thawed on ice, vortexed to mix and centrifuged for $10 \mathrm{~min}$ at $14,000 \mathrm{rpm}$ at $4^{\circ} \mathrm{C}$ in a microfuge to float lipid and pellet any debris. Ten $\mu \mathrm{l}$ of each cleared plasma was depleted of 12 Abundant proteins using Proteome Purify 12 (R\&D Biosystems) immunodepletion resin (Krishnan et al., 2017) according to the manufacturer's instructions, and the recovered protein solution was acetoneprecipitated by combining with 5 volumes of acetone and storing at $-20^{\circ} \mathrm{C}$ for $16 \mathrm{~h}$. This process is designed to remove quantities of $\alpha 1$-Acid Glycoprotein, $\alpha 1$-Antitrypsin, $\alpha 2$-Macroglobulin, Albumin, Apolipoprotein A-I, Apolipoprotein A-II, Fibrinogen, Haptoglobin, IgA, IgG, IgM and Transferrin, but also leaves residual amounts of these proteins, as the removal is not quantitative (not shown). Protein precipitate was recovered by centrifuging at $14,000 \mathrm{rpm}$ at $4^{\circ} \mathrm{C}$ for $30 \mathrm{~min}$, washed with $50 \%$ acetone, centrifuged again and dissolved in $0.1 \mathrm{M}$ Tris- $\mathrm{HCl}, \mathrm{pH}$ 7.5 with $4 \%$ SDS. Protein concentrations were measured using a
BCA microassay and all samples adjusted to the lowest common concentration, $0.7 \mu \mathrm{g} / \mu \mathrm{l}$.

\section{Filter-Assisted Sample Preparation Combined With TMT Labeling (iFASP) for Proteomic Analysis}

Filter-assisted sample preparation (Wisniewski, 2017) was carried out with the 12 AP samples and 12 controls, as follows: $90 \mu \mathrm{l}$ (i.e., $60 \mu \mathrm{g}$ ) of each sample was transferred to filter units (Millipore Ultrafree-MC 30,000 Nominal Molecular Weight Limit, low binding regenerated cellulose), the reducing agent TCEP was added to $10 \mathrm{mM}$, and the tubes placed on a ThermoMixer and heated to $55^{\circ} \mathrm{C}$ for $1 \mathrm{~h}$ with occasional mixing. Then, $8 \mathrm{M}$ urea in $50 \mathrm{mM}$ TEAB (UT) Buffer $(300 \mu \mathrm{l}$ ) was added, the samples inverted to mix, and buffer containing excess reducing agent and SDS was depleted from the sample by centrifuging the soluble components through the filter bed at 10,000 rpm on a microfuge at RT. When samples in the filters reached $\sim 50 \mu \mathrm{l}$, they were washed again with $300 \mu \mathrm{l}$ UT Buffer. To alkylate proteins, $50 \mathrm{mM}$ iodoacetic acid was added. The samples were mixed in the Thermomixer for $1 \mathrm{~min}$, then placed in the dark for $30 \mathrm{~min}$. One wash with UT buffer was followed by two washes with $50 \mathrm{mM}$ TEAB buffer $(200 \mu \mathrm{l}$ each). Proteomic-grade trypsin (Thermo Fisher) was dissolved in the buffer provided $(20 \mu \mathrm{l})$, then combined with $50 \mathrm{mM}$ TEAB, and $85 \mu \mathrm{l}$ of this solution was transferred to each filter to give a final trypsin:protein ratio of 1:40. The filters were sealed with Parafilm, then shaken at $600 \mathrm{rpm}$ with temperature set to $37^{\circ} \mathrm{C}$ for $18 \mathrm{~h}$.

Following trypsinization, sample volumes were measured, and 0.125 volume of each sample was removed and combined to make a "master mix" or reference standard, which was divided and transferred to three new filters. TMT labeling according to the scheme shown in Figure $\mathbf{1}$ was performed according to the manufacturer's instructions, while peptides were still in the columns, an innovation "iFASP" introduced by the Steen lab (McDowell et al., 2013) to the established FASP method (Wisniewski et al., 2009). Labeled peptides were then spun through the filters, with extraction assisted by twice adding $40 \mu \mathrm{l}$ $50 \mathrm{mM}$ TEAB, shaking at $800 \mathrm{rpm}$ for $20 \mathrm{~min}$ at $37^{\circ} \mathrm{C}$ and then spinning the filter tubes to collect labeled peptides in clean LoBind tubes. A final extraction was done using $50 \mu \mathrm{l}$ of $5 \mathrm{mM}$ $\mathrm{NaCl}$ in $50 \mathrm{mM}$ TEAB. All peptides spun through the filter were combined and the volume was reduced to $\sim 170 \mu \mathrm{l} /$ tube by vacuum centrifugation.

Samples were combined into three nine-plexes, according to the scheme shown in Figure 1. The three nine-plexes of TMTlabeled peptides were desalted using OASIS HLB columns by loading in $1 \mathrm{ml} 0.1 \% \mathrm{FA}$, washing 10 times with $1 \mathrm{ml}$, then eluted once each with 30,50 , and $70 \%$ acetonitrile and combined. ACN was removed by vacuum centrifugation. Peptides were then fractionated using SCX spin columns (Thermo Fisher Scientific), added to the columns in loading solution (LS) containing $0.1 \% \mathrm{FA} / 5 \% \mathrm{ACN}$. The flow through was kept as one fraction, then after two washes with LS, further peptides were eluted sequentially using a solution (S2) containing $0.1 \% \mathrm{FA}, 0.5 \mathrm{M} \mathrm{KCl}$ and $25 \% \mathrm{ACN}$, combined with LS at 25:75, 50:50 and 100:0 (i.e., 


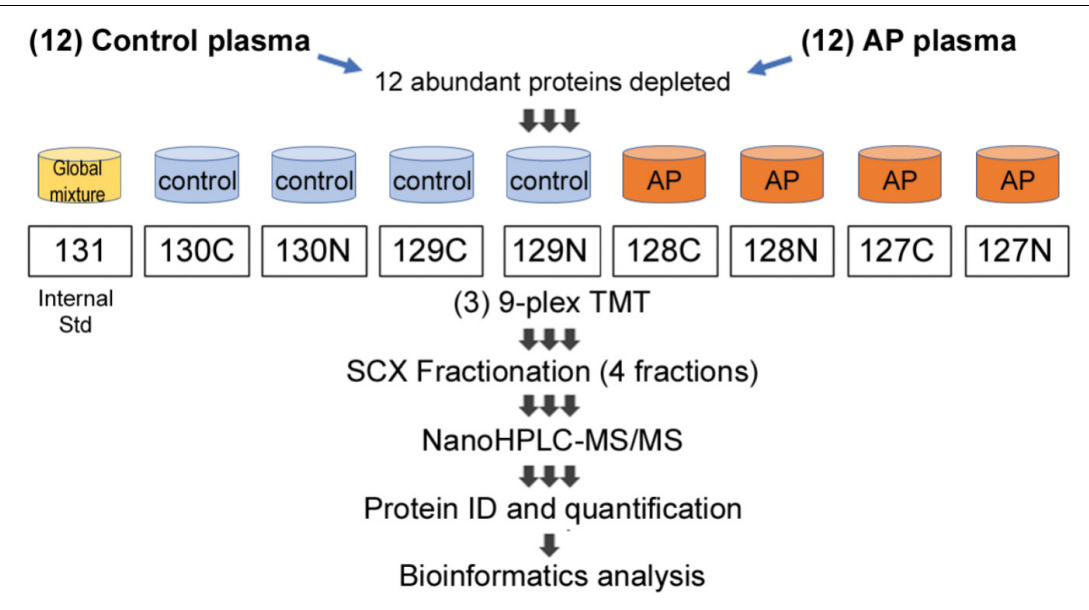

FIGURE 1 | Proteomic workflow for marker identification in alcoholic AP plasma samples. Samples were processed as described in Section "Materials and Methods" and tryptic peptides labeled with amine-reactive tandem mass tags and assembled into nine-plexes as shown. Triplicates of a master mix of all peptides were constructed, labeled with TMT-126 and incorporated into nine-plexes together with four controls and four alcoholic AP plasma samples. As there were 12 controls and 12 alcoholic AP plasma samples, three nine-plexes were needed. Each nine-plex was fractionated into four subfractions and then the 12 samples run sequentially in the mass spectrometry experiment. Data were compiled, proteins identified and quantitated using Proteome Discoverer software. Bioinformatic analysis was performed subsequently using online DAVID software and using IPA software licensed to CSMC and as described in the text.

undiluted S2). Fractions were desalted using disposable C18 spin columns and stored at $-20^{\circ} \mathrm{C}$.

\section{LC-MS/MS and Systems Analysis of TMT 9-Plexes}

The above SCX fractionation yielded four samples from each of the three 9-plex peptide mixtures. These 12 mass spectrometry samples were analyzed by high-resolution mass spectrometry as follows: fractionated peptides were separated by low-pH RPLC and analyzed by LC-MS/MS using an EASY-nLC 1000 connected to an LTQ Orbitrap Elite Mass Spectrometer (Thermo Scientific). Briefly, peptides were loaded onto a 2-cm trap column (PepMap $100 \mathrm{C} 18,75 \mu \mathrm{m}$ inner diameter, $3 \mu \mathrm{m}$ particles, $100 \AA$ pore size) and separated by a $50-\mathrm{cm}$ EASY-Spray column (PepMap RSLC $C_{18}, 75 \mu \mathrm{m}$ inner diameter, $2 \mu \mathrm{m}$ particles, $100 \AA$ pore size) heated to $55^{\circ} \mathrm{C}$. For low-pH RPLC separation, the mobile phase consisted of $0.1 \%$ FA in water (phase A) or acetonitrile (phase B). The LC gradient was 4-24\% B over $200 \mathrm{~min}, 24-$ $50 \% \mathrm{~B}$ over $20 \mathrm{~min}$, and $50-100 \% \mathrm{~B}$ over $5 \mathrm{~min}$ at a flow rate of $150 \mathrm{~nL} / \mathrm{min}$, followed by $100 \% \mathrm{~B}$ over $15 \mathrm{~min}$ at a flow rate of $300 \mathrm{~nL} / \mathrm{min}$. Mass spectra were acquired in a data-dependent manner, selecting up to 15 most abundant precursor ions for higher-energy collisional dissociation. The mass resolution for precursor and fragment ions were set at 120,000 and 60,000, respectively. The isolation width was set to 1.5 and the normalized collision energy was set to 40. Pierce BSA Protein digest was used as standard for mass spec calibration. All reagents used were mass spectrometry grade. Data were analyzed using Proteome Discoverer (v2.1), with FDR set to $1 \%$ and proteins identified on the basis of at least two unique peptides. Proteins identified were quantitated as the average of four triplicate values generated between individual patients and controls, given as the ratio patient/control as a fold change of patient to control. Student's $t$-test was performed and $P$-value of 0.05 taken as an indicator of significance. Further systems analysis was also carried out by analyzing data using IPA ${ }^{1}$ (IPA, QIAGEN, Inc.). IPA was performed by inputting a list of proteins elevated and reduced, with associated fold-changes.

\section{Human Pancreatic Acini and Pancreatic Tissues}

Human acini preparations and human pancreatic tissues were used in this study. Primary human pancreatic acini were obtained from cadaveric pancreatic tissues from organ donors. Briefly, pancreata were digested at City of Hope (Duarte, CA, United States) to separate islets from acinoductal trees, as described (Qi et al., 2015). The resulting acini were transferred to Cedars-Sinai Medical Center (Los Angeles, CA, United States) and further prepared and characterized as previously described (Lugea et al., 2017). The study was performed in accordance with regulations and protocols approved by the Institutional Review Boards of the Beckman Research Institute of the City of Hope and the Cedars-Sinai Medical Center (IRB Pro00032114). Briefly, normal pancreatic tissue samples were obtained from a deceased organ donor and by surgical resection from a chronic pancreatitis patient following approved institutional review board procedures (Cedars-Sinai Medical Center, institutional review board Pro00034086). Resected pancreas tissues were examined at the Surgical Pathology Department. Normal tissue samples were collected and snap frozen for subsequent analysis or formalin fixed for histological examination to ensure normal tissue morphology. Similar procedures were followed for tissue sections from cadaveric pancreata.

Acini and pancreatic tissue samples were homogenized in RIPA buffer containing $50 \mathrm{mmol} / \mathrm{L}$ Tris (pH 7.4), $150 \mathrm{mmol} / \mathrm{L}$

\footnotetext{
${ }^{1}$ https://www.qiagenbioinformatics.com/products/ingenuity-pathway-analysis/
} 
$\mathrm{NaCl}, 1 \%$ deoxycholic acid, 1\% Triton X-100, 0.1\% SDS and a mixture of protease and phosphatase inhibitors (Roche Applied Science, Basel, Switzerland). Protein extracts were resolved by Western Blot Analysis as indicated below.

\section{Immunoprecipitation and Western Blot Analysis}

To analyze transketolase expression in human pancreas tissue and acinar cells, samples of protein $(20 \mu \mathrm{g} / \mathrm{lane})$ were run in Novex WedgeWell 4-20\% Tris-glycine SDS-PAGE minigels (Invitrogen). Proteins were separated by electrophoresis and subsequently transferred to nitrocellulose membranes using the XCell SureLock mini electrophoresis cell (Invitrogen). Membranes were blocked with 5\% non-fat dry milk in TBS-T and incubated first with a rabbit anti-transketolase primary antibody (sc-67120) from Santa Cruz Biotechnology (diluted 1:400 in TBS-T, overnight at $4^{\circ} \mathrm{C}$ ), and then with an HRP-conjugated, anti-rabbit secondary antibody (Cell Signaling Technologies), at 1:5000 for $1 \mathrm{~h}$ at RT and developed using Pierce SuperSignal West Pico chemiluminescence substrate (Thermo Fisher) on a Syngene PXi6 chemiluminescence documentation instrument with GeneSys software. Protein loading control was estimated using an antibody against $\beta$-actin (\#A1978, Sigma-Aldrich). The molecular weight of transketolase $(68 \mathrm{kDa})$ was estimated using Precision Plus dual color protein markers (Bio-Rad). To analyze transketolase content of plasma, control or AP patient plasma samples previously analyzed by quantitative proteomics (chosen at random) were immunoprecitated using the Catch and Release (v.2.0) Reversible Immunoprecipitation System (Millipore), according to the manufacturer's instructions. Input protein per reaction included $11 \mu \mathrm{l}$ of plasma $(\sim 600 \mu \mathrm{g}$ protein) and $20 \mu \mathrm{l}$ (i.e., $4 \mu \mathrm{g}$ ) of antibody sc-67120 in $500 \mu \mathrm{l}$ of dilution/wash solution. Incubation was carried out overnight at $4^{\circ} \mathrm{C}$, and antibody capture effected using protein $\mathrm{A}$-decorated beads. After washing three times, beads were extracted with gel-loading buffer, and captured transketolase protein was analyzed by SDS-PAGE and Western blot analysis as described above.

\section{RESULTS}

\section{Clinical Data}

Blood samples were obtained from patients admitted to the hospital with a diagnosis of AP and an onset of the disease within the last $72 \mathrm{~h}$. The diagnosis of AP was made based on typical abdominal pain, at least threefold elevated levels of serum amylase, and typical radiological findings. Two of three criteria were needed to make a diagnosis of AP, and peripheral blood samples were drawn on admission. The severity of AP was evaluated at $48 \mathrm{~h}$ after presentation.

For this study, we tested 12 human AP plasma samples. The primary selection criteria were elevated LDH (>400 IU/L) (see Table 1) and a history of alcohol abuse as an etiologic factor. Patients with age $<20$ or $>70$ years-old were excluded. Age and sex matched healthy subjects $(n=12)$ without previous medical history were enrolled into the control group. Selected AP patients were all males with alcohol as potential etiologic factor (according to the patients self-reporting of alcohol abuse). As indicated in Table 2, the average age of AP patients was 40.3 year $($ median $=39.0)$; LDH levels, $1172.6 \mathrm{IU} / \mathrm{L}$; APACHE II score, 5.4; IMRIE score, 3.5; and MODS score, 3.3. Average duration of treatment was 15.2 days (median $=16.5$ ). Complications included: IAP $(27 \mathrm{mmHg})$ in one AP patient that died during hospitalization; another patient had MODS, heart failure and renal insufficiency; and two patients had diabetes. No surgical interventions were needed; one patient required ERCP, and two patients required ultrasound guided drainage.

\section{Proteomic Analysis Reveals Altered Levels of Several Proteins in Plasma From AP Patients Compared to Controls}

The experimental design schematically illustrating the composition of the multiplex proteomic sample labeling with TMT and assembly into three nine-plexes is shown in Figure 1. Tandem mass spectrometry and Proteome Discoverer analysis identified 4217 peptides and 285 proteins. Many proteins were changed in the AP patients relative to controls, as shown by mean values of fold change returned by the analysis. To generate a list of proteins increased or decreased, up to four determined values were possible for each protein (i.e., one from each of four fractions). The top proteins (by average fold change) as shown in Figure 2A, often elevated in all four samples, were analyzed by $t$-test. Fold-changes of 1.5 -fold or more or 0.67 -fold or less were included, and we report here only those for which $P=0.05$ or less as a measure of statistical significance (Table 2). A separate graphic in Figure 2B also depicts these proteins in a circular fashion to highlight those with the highest elevated levels.

TABLE 1 | Acute pancreatitis severity scores*.

\begin{tabular}{|c|c|c|c|c|c|c|}
\hline & Number of patients & Mean & Standard deviation & Median & Minimum & Maximum \\
\hline $\mathrm{LDH}(\mathrm{U} / \mathrm{L})$ & 12 & 1172.6 & 735.6 & 1077.5 & 420.0 & 3028.0 \\
\hline IMRIE & 12 & 3.5 & 1.8 & 3.5 & 1.0 & 6.0 \\
\hline MODS & 12 & 3.3 & 2.7 & 3.0 & 0 & 10.0 \\
\hline
\end{tabular}

*For each participant, the scores were calculated at $48 \mathrm{~h}$ after presentation. 
TABLE 2 | Plasma markers of ethanolic AP from quantitative proteomics.

\begin{tabular}{|c|c|c|c|c|c|c|}
\hline Accession & Name/gene name & MW (kDa) & Unique peptides & Coverage (\%) & Avg fold & $t$-Test ( $P$-value) \\
\hline \multicolumn{7}{|c|}{ Proteins increased in alcoholic AP } \\
\hline P02741 & C-reactive protein/CRP & 25 & 8 & 28 & 7.48 & 0.00 \\
\hline P29401 & Transketolase/TKT & 68 & 3 & 6 & 6.29 & 0.00 \\
\hline P02750 & Leucine-rich alpha-2-glycoprotein/LRG1 & 38 & 10 & 30 & 4.03 & 0.01 \\
\hline A0A096LPE2 & Protein SAA2-SAA4/SAA2-SAA4 & 23 & 6 & 42 & 3.96 & 0.01 \\
\hline P05109 & Protein S100-A8/S100A8 & 11 & 5 & 45 & 3.78 & 0.01 \\
\hline P06702 & Protein S100-A9/S100A9 & 13 & 3 & 25 & 3.33 & 0.05 \\
\hline P01011 & Alpha-1-antichymotrypsin/SERPINA3 & 48 & 21 & 43 & 3.10 & 0.00 \\
\hline Q08830 & Fibrinogen-like protein 1/FGL1 & 36 & 3 & 13 & 3.08 & 0.02 \\
\hline P02679 & Fibrinogen gamma chain/FGG & 51 & 28 & 58 & 3.06 & 0.02 \\
\hline P02675 & Fibrinogen beta chain/FGB & 56 & 38 & 71 & 2.96 & 0.02 \\
\hline P02763 & Alpha-1-acid glycoprotein 1/ORM1 & 23 & 5 & 35 & 2.91 & 0.01 \\
\hline P04275 & von Willebrand factor/WWF & 309 & 12 & 5 & 2.82 & 0.00 \\
\hline P01009 & Alpha-1-antitrypsin/SERPINAI & 47 & 28 & 66 & 2.71 & 0.01 \\
\hline P02671 & Fibrinogen alpha chain/FGA & 95 & 42 & 42 & 2.66 & 0.04 \\
\hline PODJI8 & Serum amyloid A-1 protein/SAA1 & 14 & 3 & 47 & 2.41 & 0.03 \\
\hline P13796 & Plastin-2/LCP1 & 70 & 8 & 15 & 2.39 & 0.03 \\
\hline Q9UK55 & Protein Z-dependent protease inhibitor/SERPINA10 & 51 & 5 & 10 & 1.98 & 0.00 \\
\hline P18428 & Lipopolysaccharide-binding protein/LBP & 53 & 6 & 14 & 1.86 & 0.01 \\
\hline P19652 & Alpha-1-acid glycoprotein 2/ORM2 & 24 & 4 & 26 & 1.81 & 0.05 \\
\hline P07195 & L-Lactate dehydrogenase B chain/LDHB & 37 & 2 & 11 & 1.79 & 0.01 \\
\hline P00740 & Coagulation factor IX/F9 & 52 & 8 & 17 & 1.78 & 0.00 \\
\hline P04040 & Catalase/CAT & 60 & 8 & 16 & 1.76 & 0.00 \\
\hline P08571 & Monocyte differentiation antigen CD14/CD14 & 40 & 4 & 9 & 1.70 & 0.00 \\
\hline Q06033 & Inter-alpha-trypsin inhibitor heavy chain H3/ITIH3 & 100 & 13 & 16 & 1.69 & 0.03 \\
\hline P02748 & Complement component C9/C9 & 63 & 23 & 37 & 1.57 & 0.05 \\
\hline P05155 & Plasma protease C1 inhibitor/SERPING1 & 55 & 14 & 26 & 1.54 & 0.01 \\
\hline P07998 & Ribonuclease pancreatic/RNASEI & 18 & 2 & 19 & 1.53 & 0.03 \\
\hline P00736 & Complement C1r subcomponent/C1R & 80 & 17 & 27 & 1.53 & 0.02 \\
\hline P01019 & Angiotensinogen/AGT & 53 & 8 & 18 & 1.50 & 0.02 \\
\hline \multicolumn{7}{|c|}{ Proteins decreased in alcoholic AP } \\
\hline P80108 & Phosphatidylinositol-glycan-specific phospholipase D/GPLD1 & 92 & 11 & 13 & 0.63 & 0.01 \\
\hline P02775 & Platelet basic protein/PPBP & 14 & 3 & 26 & 0.61 & 0.02 \\
\hline Q9UGM5 & Fetuin-B/FETUB & 42 & 6 & 16 & 0.60 & 0.02 \\
\hline P02787 & Serotransferri n/T F & 77 & 48 & 58 & 0.54 & 0.02 \\
\hline P05154 & Plasma serine protease inhibitor/SERPINA5 & 46 & 5 & 14 & 0.47 & 0.00 \\
\hline P02768 & Serum albumin/ALB & 69 & 76 & 87 & 0.46 & 0.02 \\
\hline
\end{tabular}

\section{Gene Ontology and Ingenuity Pathway Analysis (IPA)}

Gene Ontology analysis was performed using the 31 significantly elevated or the six decreased proteins as input (Table 2). Thus, according to analysis using the Database for Annotation, Visualization and Integrated Discovery (DAVID) software (Huang et al., 2009a,b) version 6.8, the major gene ontology categories (Biological Processes, Cellular compartments and Molecular Functions) and Kyoto Encyclopedia of Genes and Genomes (KEGG) pathways detected are described in Figure 3.

Notable biological processes associated with proteins elevated in AP samples include platelet degranulation, acute phase response, blood coagulation, platelet activation, and negative regulation of endopeptidase activity. Notable cellular compartments associated with the elevated proteins include exosomes, extracellular space, and platelet alpha granule lumen. The most closely associated molecular function with the proteins elevated in AP was serine-type endopeptidase inhibitor activity, due to the presence of multiple SERPINs, ITIH3 and AGT, a non-inhibitory member of the SERPIN family (Heit et al., 2013). ITIH3 was recently reported as a PDAC serum biomarker (Liu et al., 2017).

According to DAVID analysis, nearly all (29 of 31 ) the increased proteins have been associated with or detected in the cellular compartment of exosomes. Exosomes are secreted particles initiated by invagination of late endosomal boundary membrane and packaged by the ESCRT pathway into multivesicular bodies. Overall, whereas the proteins increased in ethanolic AP plasma corresponded to a wide range of blood functions, compartments and processes, including ordinary 


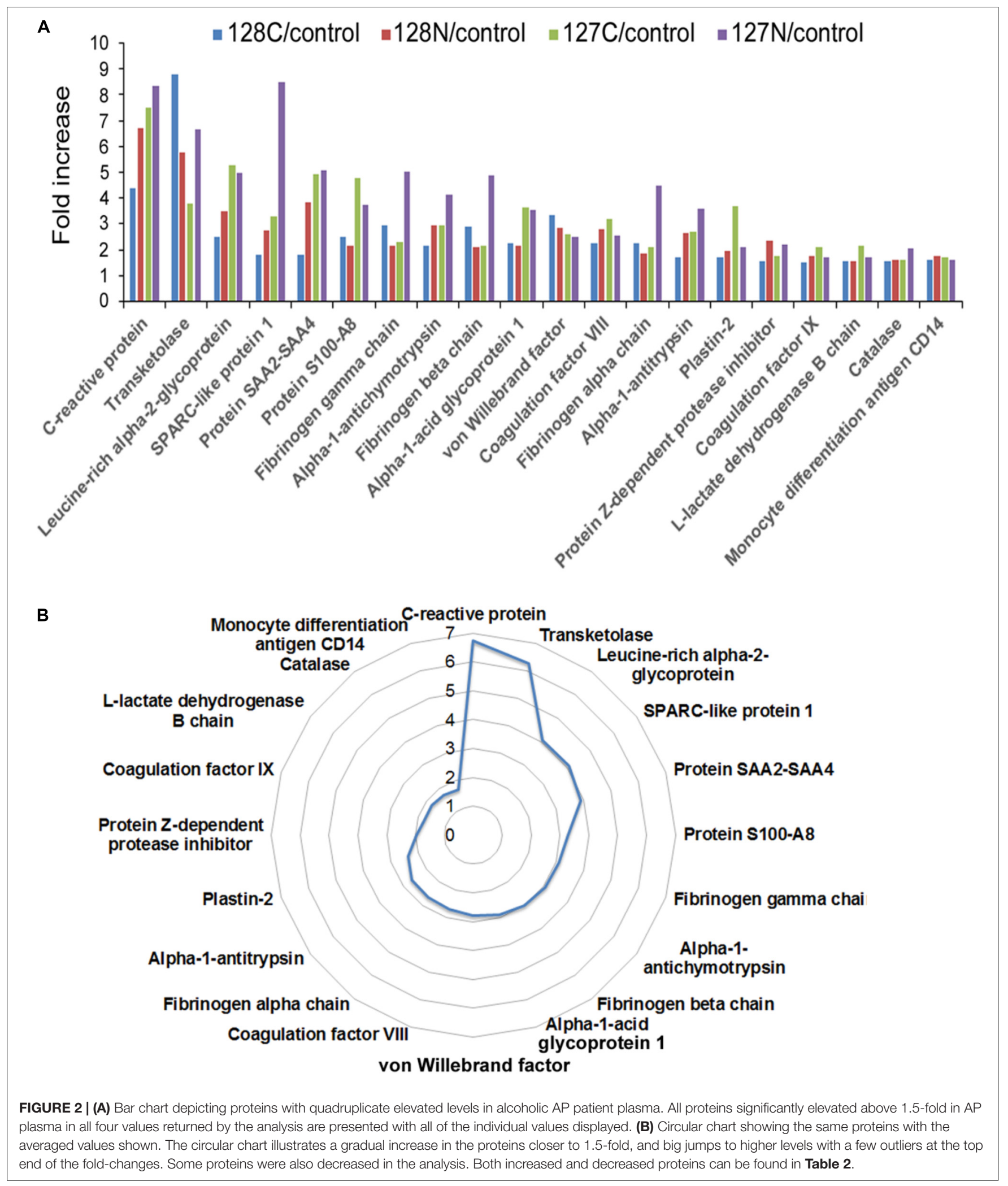

complement, fibrinolysis and blood coagulation components normally secreted to the extracellular space, there is also evidence of pathologic issues such as platelet degranulation and the acute phase response, in addition to both innate and adaptive immunity. We note that some of the proteins differentially elevated or decreased in our TMT-based quantitative analysis 


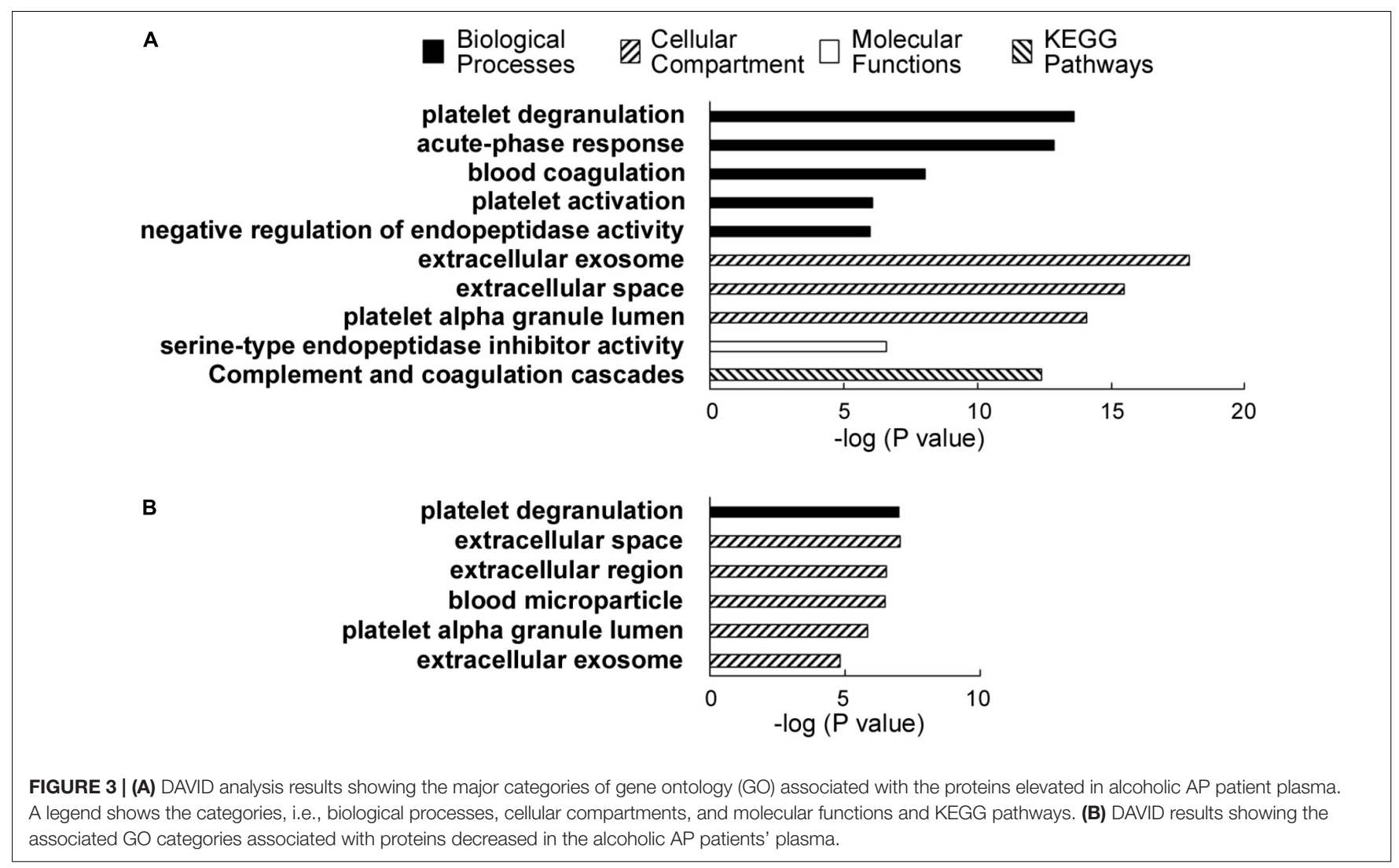

are among the 12 depleted proteins. These include $\alpha 1$ antitrypsin/SERPINA1, Albumin and Transferrin. However, as these proteins were depleted consistently from all samples, it is unlikely that these differences arose from the depletion process used in our sample preparation workflow. A recent article that used the same abundant plasma protein removal procedure had a similar conclusion (Krishnan et al., 2017).

We further extended our systems analysis of the ensemble of proteins elevated and reduced in alcoholic AP by IPA analysis. Proteins (Uniprot IDs) were input into the analysis together with up or down fold-changes. Results of this analysis are shown in Figure 4. IPA analysis predicted that many of the acute phase proteins are synthesized via an upstream signal transduction network involving cytokines such as TNF $\alpha$, IL-1, IL-6 and OSM, and key transcription factors such as STAT3 (see Figure 4A). Other transcription factors such as NF- $\mathrm{B}, \mathrm{AP}-1$ and NF-IL6, and nuclear factors such as corticosteroid-GCR also play important roles in the acute phase signaling pathway network (data not shown). These signal transduction pathways initiated in hepatocytes and other cells induce many downstream events including chemotaxis, binding and invasion of inflammatory cells, as shown schematically in Figure 4B. Elevation of genes such as angiotensinogen and apolipoproteins E and C2 were also predictive of upstream activation of LXR/RXR signaling (not shown). A summary of the associated canonical pathways and $P$-values as a measure of their relative statistical significance is shown in Figure 4C. In AP, many other cytokines, chemokines, and signaling molecules may also be involved in triggering various responses which systematically account for SIRS and organ failure (Dambrauskas et al., 2010). We anticipate that insights into the upstream pathways triggering AP such as those produced by IPA analysis will give rise to targeted treatments for AP.

\section{Validation of Selected Proteins Found Elevated in Plasma From AP Patients Compared to Controls}

Among the distinct proteins found elevated in the AP samples (shown in Figure 2 and Table 2), CRP had the highest foldchange, measured as 7.5-fold higher in AP than control samples. As mentioned above, CRP has been studied and found to be an excellent indicator of AP severity, although it can be elevated in other inflammatory conditions (Komolafe et al., 2017). Its use in the clinic is increasing due to availability of an inexpensive test (Meher et al., 2015), although its diagnostic accuracy may depend on the timing and the clinical utility of CRP or other biomarkers for prediction remains uncertain (Komolafe et al., 2017). Whereas most of the proteins we found elevated in AP are secreted proteins, the second highest protein, transketolase is an intracellular enzyme. Leucine-rich $\alpha 2$ glycoprotein (LRG1) has been identified as a marker of granulocyte differentiation, is involved in neovascularization by altering TGF $\alpha$ signaling at endothelial cells (Wang et al., 2013), and has recently been found elevated in plasma associated with distinct cancers including pancreatic (Capello et al., 2017). SPARC-like protein 1, also 
A

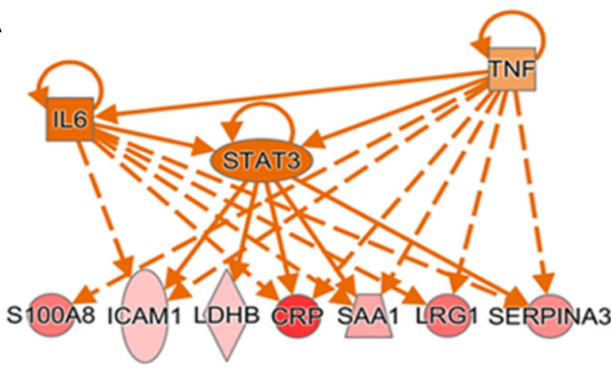

B

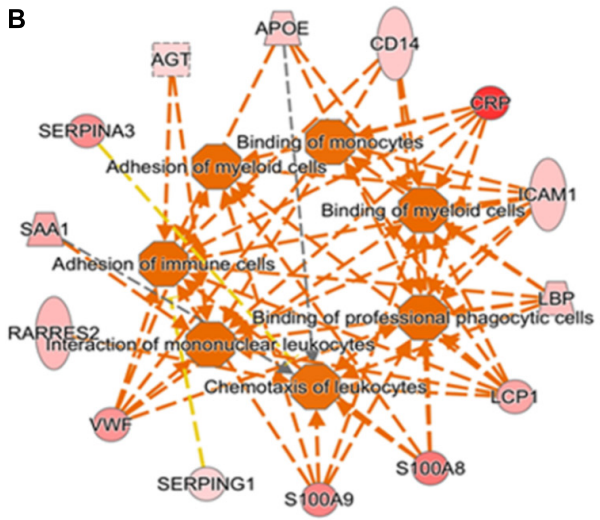

C

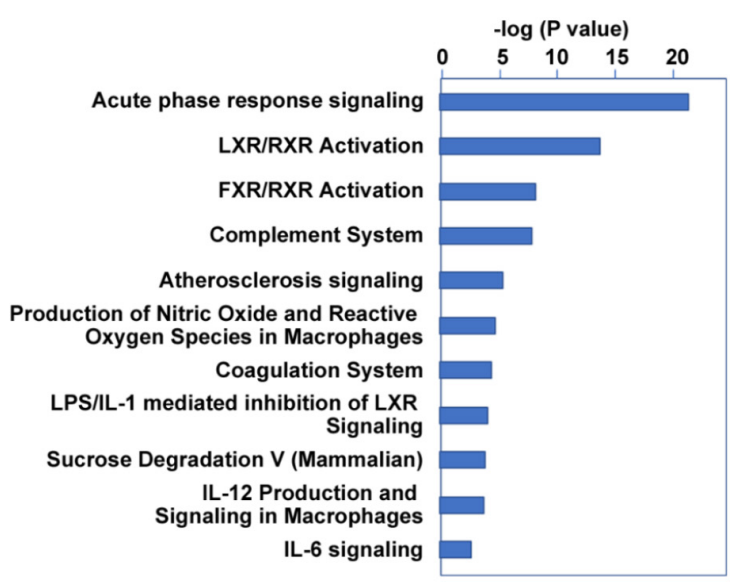

FIGURE 4 | Ingenuity pathway analysis cascading upstream cytokine and transcription factor regulatory pathways (A) and downstream pathobiologic effectors (B) predicted from our proteomic dataset. Dark orange implies a predicted mediator or process. Red intensity shows expression level. (C) Major canonical pathways associated with the gene set.
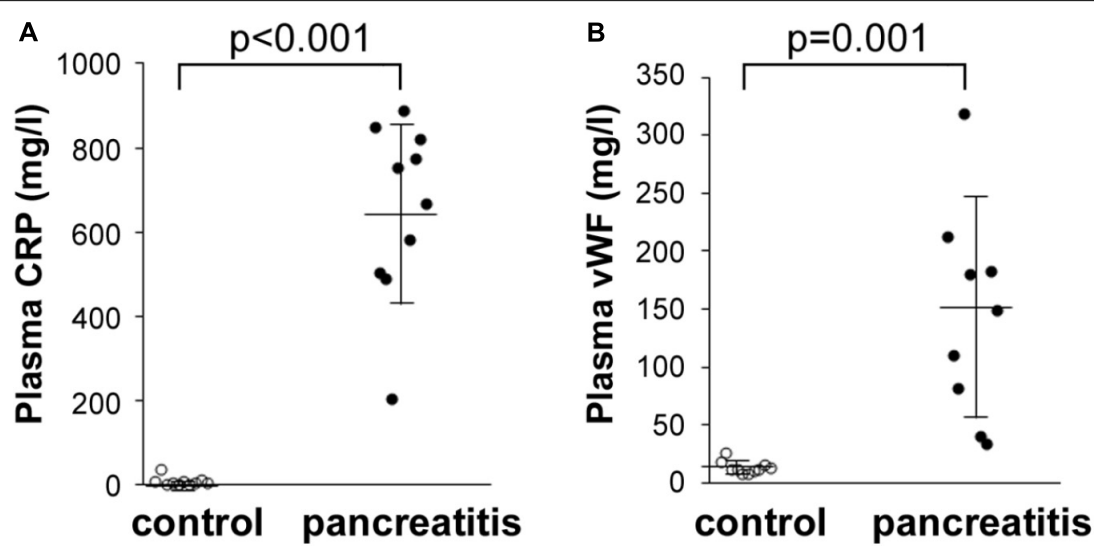

FIGURE 5 | Luminex determination of (A) CRP and (B) VWF in control and alcoholic AP patient plasma samples. Samples were thawed, diluted 40,000-fold as instructed by the manufacturer, and processed further according to instructions. Readings were taken from duplicate measurements, and controls versus alcoholic AP patient plasma results graphed with the mean and standard deviation shown by the horizontal lines with error bars; $n=9-10$. Statistical significance was evaluated by Student's $t$-test. $P$-value for CRP measurement in AP patients was $<0.001$. $P$-value for $v W F$ was $=0.001$.

known as HEVIN, is described as a matricellular protein, which may have positive and/or negative roles in distinct cancers (Gagliardi et al., 2017). S100A8, or calprotectin is a small calciumbinding protein often associated with immune cells (Gebhardt et al., 2006). Plastin-2 or Plastin-L/LCP1 is an actin-binding protein associated with immune cells and may be elevated in epithelial cells undergoing tumorigenesis (Shinomiya, 2012). Among the other elevated proteins, one that has been widely hypothesized to play distinct roles in pancreatic diseases is angiotensinogen (Skipworth et al., 2011).

We also performed additional laboratory tests to reevaluate the levels of CRP, as well as another acute phase protein found elevated in the AP patients' sera by quantitative mass spectrometry, vWF. To achieve this, we performed Luminex assays in a number of the same plasma samples analyzed by quantitative LC-MS/MS. Shown in Figure 5A, CRP levels in all 
the AP plasma samples were dramatically elevated compared with those in control plasma samples. Similarly, vWF levels were also elevated, but to a lesser extent than CRP (Figure 5B). These data indicate that a highly sensitive and exacting test such as Luminex provides a similar series of elevated levels as that reported by TMT labeling/LC-MS/MS, i.e., CRP > vWF. These data thereby independently validate our finding of uniform elevation of these two acute phase proteins in the AP patient plasma samples analyzed.

Elevated plasma transketolase (TKT), to our knowledge has not been previously reported as an AP biomarker. This enzyme is normally not secreted, although it is expressed in pancreas and may be released from damaged pancreatic tissue. Here, we also performed Western blot analysis to detect TKT expression. We first showed that a commercial antibody was successful to detect TKT in samples of human pancreas tissue and isolated human acinar cells (Figure 6). These data show the efficacy of the antibody and the substantial expression in pancreas. We next used the antibody to isolate elevated plasma TKT by immunoprecipitation from a subset of proteomically analyzed AP plasma samples. Thus, full length TKT was immunoprecipitated from two control and two AP samples. Results of Western blot analysis carried out with these immunoprecipitated samples, shown in Figure 6 demonstrate that, whereas there is no detectable TKT in control plasma immunoprecipitates, bands selectively appear in AP samples. These data provide further evidence which substantiates our finding of increased TKT in the AP patients' plasma by mass spectrometry.

\section{DISCUSSION}

The presence of elevated levels of proteins identified in this study, including a significant number of acute phase response proteins may be indicative of distinct disease processes and risk for severe inflammatory disease complications and cancer. Further study is needed to determine the specificity and selectivity of these proteins as biomarkers in alcoholic AP. Acute phase proteins appear to be well-suited to confirm the presence of systemic inflammatory process in AP and individual proteins such as CRP will likely continue to be used clinically. However, these proteins may also be elevated in other disease processes, and therefore they lack specificity. More novel is our finding of elevated TKT, the cellular source of which is unknown. Measurement of erythrocyte TKT activity has been used for many years as a measure of thiamine deficiency (Thomson et al., 2010). Also, erythrocyte abnormalities and hemolysis are known to occur in alcoholics (Ballard, 1997). Further, another study found that TKT protein can be diminished in hemolysates from alcoholic subjects (Takeuchi et al., 1988). Taken together, these considerations suggest erythrocytes as a possible source of elevated plasma TKT in alcoholic AP patients.

However, our previous study undertaken with proteomic analysis of normal human pancreas tissue also indicate that TKT is highly expressed in pancreas (Lugea et al., 2017). Thus, excess TKT in plasma could also be evidence of pancreas damage. Further studies will be needed to evaluate whether TKT elevation

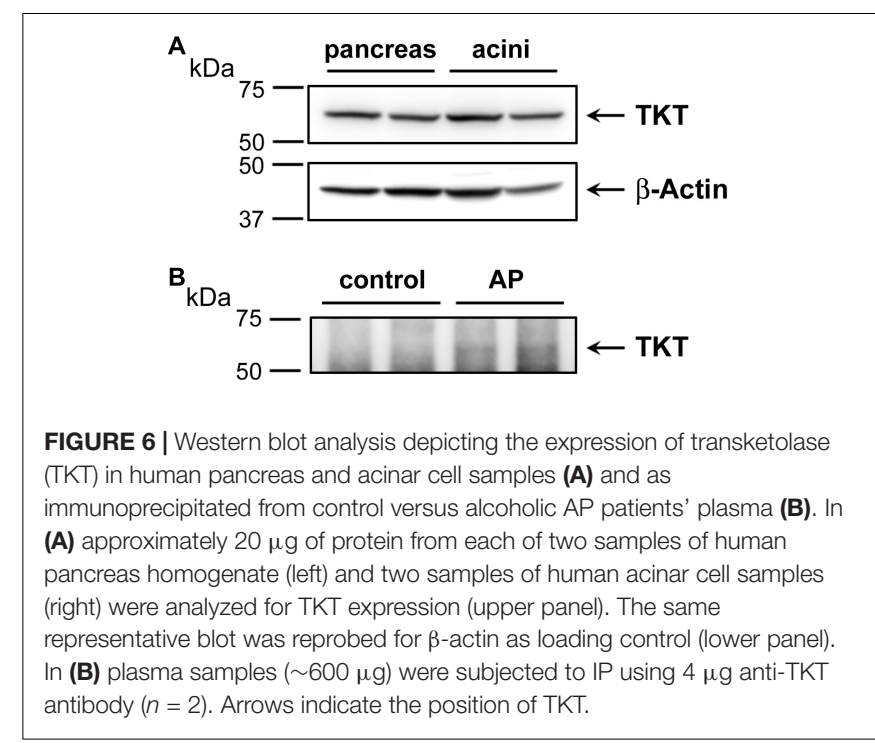

is unique to alcoholic AP, is found more generally elevated in $\mathrm{AP}$, or is useful as a measure of predicted severity in these or other patients. An important metabolic role was recently ascribed to TKT to promote pancreatic cancer development through conversion of fructose to nucleotides for anabolic metabolism (Liu et al., 2010). Elevation of LRG1, SPARCL1, S100A8, Plastin-2 may be of special significance in a small but significant percentage of patients which have increased risk for prolonged development of a disease spectrum that connects AP, RAP, and chronic pancreatitis with emergence of pancreatic cancer. Pancreatic cancer exhibits profound alterations in neovascularization, which likely precede and may even be pivotal in tumor initiation in the context of CP. Thus, findings reported here may have added significance in increasing our understanding of the poorlyunderstood links between pancreatitis and pancreatic cancer.

The use of IPA as an adjunct to genomic and proteomic investigations is rising steadily and has been included in over 2000 published papers in PubMed, 42 of which touch upon some aspect of pancreas or pancreatic research. IPA relies on a sophisticated, comprehensive manually curated information base, a strength of which is its ability to factor in whether the level of each gene increases or decreases. This allows data to be integrated, interpreted, and displayed in the context of previously documented biological pathways. In one study, different oncogenes were introduced into a normal ductal cell background, and IPA was used to understand which tumorigenic pathways were initiated in the cells (Chang et al., 2013). More recently, IPA was used to interpret effects of epigenetic reprogramming in Mist1-/- mice (Mehmood et al., 2014). Here, IPA showed that many of the proteins elevated during alcoholic pancreatitis are those of the acute phase response. Beyond this, some of the key transcription factors and chemokines/cytokines involved were pinpointed, giving indications as to which pathways are activated and might be targeted in future studies, or even therapeutically. It has become clear that incorporating IPA and other bioinformatic approaches into studies that generate a set of genes or proteins has great potential to enhance our 
understanding of how normal biology is disrupted in disease processes.

\section{CONCLUSION}

Given the wide range of outcomes in AP, and the failure of clinical scoring systems to adequately prepare caregivers for different outcomes in the AP disease course, much research has been dedicated to identification of stand-alone protein biomarkers which would be of value in assessing the severity of AP patients. Nevertheless, whereas several potential markers of severity including CRP have been tested to a limited extent, none have been established as standards of care for this purpose. Here, we used a shotgun proteomics approach to search for proteins elevated or decreased in a small group of alcoholic AP patients versus controls. This approach resulted in the identification of CRP as the most elevated protein in these plasma samples, which validates our approach since this is the protein most often regarded as a valuable early AP biomarker. Other increased proteins including transketolase are novel and will require further study. Some proteins were also selectively decreased in alcoholic AP samples in this study. These may also be significant in pancreas diseases as well as in various other inflammatory diseases.

\section{REFERENCES}

Aframian, D., Katzenellenbogen, M., Arad, G., Osman, F., Sayar, D., Ketzinel, M., et al. (1996). Down-regulation of human tumor necrosis factor-beta gene expression by cells with suppressive activity. Immunol. Lett. 54, 171-176. doi: 10.1016/S0165-2478(96)02668-5

Andriulli, A., Botteri, E., Almasio, P. L., Vantini, I., Uomo, G., Maisonneuve, P., et al. (2010). Smoking as a cofactor for causation of chronic pancreatitis: a meta-analysis. Pancreas 39, 1205-1210. doi: 10.1097/MPA.0b013e3181df $27 \mathrm{c} 0$

Ballard, H. S. (1997). The hematological complications of alcoholism. Alcohol Health Res. World 21, 42-52.

Capello, M., Bantis, L. E., Scelo, G., Zhao, Y., Li, P., Dhillon, D. S., et al. (2017). Sequential validation of blood-based protein biomarker candidates for earlystage pancreatic cancer. J. Natl. Cancer Inst. 109:djw266. doi: 10.1093/jnci/ djw266

Chang, Z., Li, Z., Wang, X., Kang, Y., Yuan, Y., Niu, J., et al. (2013). Deciphering the mechanisms of tumorigenesis in human pancreatic ductal epithelial cells. Clin. Cancer Res. 19, 549-559. doi: 10.1158/1078-0432.CCR12-0032

Dambrauskas, Z., Giese, N., Gulbinas, A., Giese, T., Berberat, P. O., Pundzius, J., et al. (2010). Different profiles of cytokine expression during mild and severe acute pancreatitis. World J. Gastroenterol. 16, 1845-1853. doi: 10.3748/wjg.v16. i15. \{\break\} 1845

Dufour, M. C., and Adamson, M. D. (2003). The epidemiology of alcohol-induced pancreatitis. Pancreas 27, 286-290. doi: 10.1097/00006676-200311000-0 0002

Gagliardi, F., Narayanan, A., and Mortini, P. (2017). SPARCL1 a novel player in cancer biology. Crit. Rev. Oncol. Hematol. 109, 63-68. doi: 10.1016/j.critrevonc. 2016.11.013

Gebhardt, C., Nemeth, J., Angel, P., and Hess, J. (2006). S100A8 and S100A9 in inflammation and cancer. Biochem. Pharmacol. 72, 1622-1631. doi: 10.1016/j. bcp.2006.05.017

Greer, J. B., Thrower, E., and Yadav, D. (2015). Epidemiologic and mechanistic associations between smoking and pancreatitis. Curr. Treat. Options Gastroenterol. 13, 332-346. doi: 10.1007/s11938-015-0056-9

\section{AUTHOR CONTRIBUTIONS}

RW performed the proteomic and other experiments and bioinformatic analyses, analyzed and interpreted data with all other authors and drafted the manuscript and figures. AL analyzed and interpreted data and revised the manuscript and figures. AG procured samples and clinical information, initiated the study, and revised the manuscript. SP interpreted data and revised the manuscript.

\section{FUNDING}

This work was supported by Cedars-Sinai Medical Center, Vilnius University Hospital Santaros Clinics, and NIH (Grant Nos. R01 AA019954, P50 AA11999, P01 DK098108, and U01 DK108314).

\section{ACKNOWLEDGMENTS}

We thankfully acknowledge the contributions of patients and other donors who provided blood samples for this study. We also thank the Biomarker Discovery Platform Mass Spec Core at CSMC.

Heit, C., Jackson, B. C., McAndrews, M., Wright, M. W., Thompson, D. C., Silverman, G. A., et al. (2013). Update of the human and mouse SERPIN gene superfamily. Hum. Genomics 7:22. doi: 10.1186/1479-7364-7-22

Huang, D. W., Sherman, B. T., and Lempicki, R. A. (2009a). Bioinformatics enrichment tools: paths toward the comprehensive functional analysis of large gene lists. Nucleic Acids Res. 37, 1-13. doi: 10.1093/nar/gkn923

Huang, D. W., Sherman, B. T., and Lempicki, R. A. (2009b). Systematic and integrative analysis of large gene lists using DAVID bioinformatics resources. Nat. Protoc. 4, 44-57. doi: 10.1038/nprot.2008.211

Ignatavicius, P., Gulla, A., Cernauskis, K., Barauskas, G., and Dambrauskas, Z. (2017). How severe is moderately severe acute pancreatitis? Clinical validation of revised 2012 atlanta classification. World J. Gastroenterol. 23, 7785-7790. doi: 10.3748/wjg.v23.i43.7785

Klumbiene, J., Kalasauskas, D., Petkeviciene, J., Veryga, A., and Sakyte, E. (2012). Trends and social differences in alcohol consumption during the postcommunist transition in Lithuania. ScientificWorldJournal 2012:615183. doi: 10.1100/2012/615183

Komolafe, O., Pereira, S. P., Davidson, B. R., and Gurusamy, K. S. (2017). Serum C-reactive protein, procalcitonin, and lactate dehydrogenase for the diagnosis of pancreatic necrosis. Cochrane Database Syst. Rev. 4:CD012645. doi: 10.1002/ 14651858.CD012645

Krishnan, S., Whitwell, H. J., Cuenco, J., Gentry-Maharaj, A., Menon, U., Pereira, S. P., et al. (2017). Evidence of altered glycosylation of serum proteins prior to pancreatic cancer diagnosis. Int. J. Mol. Sci. 18:E2670. doi: 10.3390/ ijms18122670

Liu, H., Huang, D., McArthur, D. L., Boros, L. G., Nissen, N., and Heaney, A. P. (2010). Fructose induces transketolase flux to promote pancreatic cancer growth. Cancer Res. 70, 6368-6376. doi: 10.1158/0008-5472.CAN-09-4615

Liu, X., Zheng, W., Wang, W., Shen, H., Liu, L., Lou, W., et al. (2017). A new panel of pancreatic cancer biomarkers discovered using a mass spectrometry-based pipeline. Br. J. Cancer 117, 1846-1854. doi: 10.1038/bjc.20 17.365

Lugea, A., Waldron, R. T., Mareninova, O. A., Shalbueva, N., Deng, N., Su, H. Y., et al. (2017). Human pancreatic acinar cells: proteomic characterization, physiologic responses, and organellar disorders in ex vivo pancreatitis. Am. J. Pathol. 187, 2726-2743. doi: 10.1016/j.ajpath.2017.08.017 
McDowell, G. S., Gaun, A., and Steen, H. (2013). iFASP: combining isobaric mass tagging with filter-aided sample preparation. J. Proteome Res. 12, 3809-3812. doi: $10.1021 / \mathrm{pr} 400032 \mathrm{~m}$

Meher, S., Mishra, T. S., Sasmal, P. K., Rath, S., Sharma, R., Rout, B., et al. (2015). Role of biomarkers in diagnosis and prognostic evaluation of acute pancreatitis. J. Biomark. 2015:519534. doi: 10.1155/2015/519534

Mehmood, R., Varga, G., Mohanty, S. Q., Laing, S. W., Lu, Y., Johnson, C. L., et al. (2014). Epigenetic reprogramming in Mist1(-/-) mice predicts the molecular response to cerulein-induced pancreatitis. PLoS One 9:e84182. doi: 10.1371/ journal.pone.0084182

Pandol, S. J., Periskic, S., Gukovsky, I., Zaninovic, V., Jung, Y., Zong, Y., et al. (1999). Ethanol diet increases the sensitivity of rats to pancreatitis induced by cholecystokinin octapeptide. Gastroenterology 117, 706-716. doi: 10.1016/ S0016-5085(99)70465-8

Papachristou, G. I., Muddana, V., Yadav, D., O’Connell, M., Sanders, M. K., Slivka, A., et al. (2010). Comparison of BISAP, Ranson's, APACHE-II, and CTSI scores in predicting organ failure, complications, and mortality in acute pancreatitis. Am. J. Gastroenterol. 105, 435-441; quiz 442. doi: 10.1038/ajg.20 09.622

Qi, M., McFadden, B., Valiente, L., Omori, K., Bilbao, S., Juan, J., et al. (2015). Human pancreatic islets isolated from donors with elevated HbAlc levels: islet yield and graft efficacy. Cell Transplant. 24, 1879-1886. doi: 10.3727/ 096368914 X683548

Ritchie, R. F., Palomaki, G. E., Neveux, L. M., Navolotskaia, O., Ledue, T. B., and Craig, W. Y. (1999). Reference distributions for the negative acute-phase serum proteins, albumin, transferrin and transthyretin: a practical, simple and clinically relevant approach in a large cohort. J. Clin. Lab. Anal. 13, 273-279. doi: 10.1002/(SICI)1098-2825(1999)13:6<273::AID-JCLA4>3.0.CO;2-X

Shinomiya, H. (2012). Plastin family of actin-bundling proteins: its functions in leukocytes, neurons, intestines, and cancer. Int. J. Cell Biol. 2012:213492. doi: $10.1155 / 2012 / 213492$

Skipworth, J. R., Szabadkai, G., Olde Damink, S. W., Leung, P. S., Humphries, S. E., and Montgomery, H. E. (2011). Review article: pancreatic renin-angiotensin systems in health and disease. Aliment. Pharmacol. Ther. 34, 840-852. doi: $10.1111 /$ j.1365-2036.2011.04810.x
Takeuchi, T., Jung, E. H., Nishino, K., and Itokawa, Y. (1988). Western blotting assay of transketolase concentration in human hemolysates. Anal. Biochem. 168, 470-475. doi: 10.1016/0003-2697(88)90345-4

Tenner, S., Baillie, J., DeWitt, J., Vege, S. S., and American College of Gastroenterology. (2013). American college of gastroenterology guideline: management of acute pancreatitis. Am. J. Gastroenterol. 108, 1400-1415; 1416. doi: 10.1038/ajg.2013.218

Thomson, A. D., Marshall, E. J., and Guerrini, I. (2010). Biomarkers for detecting thiamine deficiency-improving confidence and taking a comprehensive history are also important. Alcohol Alcohol. 45:213. doi: 10.1093/alcalc/agq004

Wang, X., Abraham, S., McKenzie, J. A. G., Jeffs, N., Swire, M., Tripathi, V. B., et al. (2013). LRG1 promotes angiogenesis by modulating endothelial TGF-beta signalling. Nature 499, 306-311. doi: 10.1038/nature12345

Wisniewski, J. R. (2017). Filter-aided sample preparation: the versatile and efficient method for proteomic analysis. Methods Enzymol. 585, 15-27. doi: 10.1016/bs. mie.2016.09.013

Wisniewski, J. R., Zougman, A., and Mann, M. (2009). Combination of FASP and StageTip-based fractionation allows in-depth analysis of the hippocampal membrane proteome. J. Proteome Res. 8, 5674-5678. doi: 10.1021/pr900748n

Wu, B. U., Batech, M., Quezada, M., Lew, D., Fujikawa, K., Kung, J., et al. (2017). Dynamic measurement of disease activity in acute pancreatitis: the pancreatitis activity scoring system. Am. J. Gastroenterol. 112, 1144-1152. doi: 10.1038/ajg. 2017.114

Conflict of Interest Statement: The authors declare that the research was conducted in the absence of any commercial or financial relationships that could be construed as a potential conflict of interest.

Copyright (c) 2018 Waldron, Lugea, Gulla and Pandol. This is an open-access article distributed under the terms of the Creative Commons Attribution License (CC BY). The use, distribution or reproduction in other forums is permitted, provided the original author(s) and the copyright owner(s) are credited and that the original publication in this journal is cited, in accordance with accepted academic practice. No use, distribution or reproduction is permitted which does not comply with these terms. 